\title{
LE VOCABULAIRE DES MARAIS ET MARÉCAGES DANS L'HISTORIOGRAPHIE LATINE DE LA RÉPUBLIQUE ROMAINE ET DU PRINCIPAT
}

\section{THE VOCABULARY FOR MARSHES AND SWAMPS IN THE LATIN HISTORIOGRAPHY OF THE ROMAN REPUBLIC AND THE PRINCIPATUS}

\author{
MARTINE CHASSIGNET \\ chassign@,unistra.fr \\ UNIVERSITE DE STRASBOURG ${ }^{1}$ \\ [RECIBIDO: 01/03/ 2019; ACEPTADO: 20/05/2019] \\ http://dx.doi.org/10.25267/Riparia.2019.v5.05
}

\begin{abstract}
RÉSUMÉ
L'étude du vocabulaire afférent aux marais et marécages chez les historiens latins de la République romaine et du Principat montre que les termes que ces auteurs utilisent servent à préciser le cadre dans lequel se déroulent certains événements, essentiellement militaires. Le choix de ces vocables est en partie subordonné au fait que l'histoire à Rome n'est pas une science mais un genre littéraire, le plus souvent au service d'une idéologie ou d'une volonté de propagande. Elle servit de modèle à la fin de la République et sous le Principat.
\end{abstract}

MOTS-ClÉ: marais ; marécage ; intérêt économique ; intérêt stratégique ; dramatisation ; idéologie ; apologie.

1 Professeur émérite de l'Université de Strasbourg, Centre d'Analyse des Rhétoriques Religieuses de l'Antiquité (CARRA EA 3094).

M. Chassignet, «Le vocabulaire des marais et marécages dans l'historiographie latine de la République romaine et du Principat », RIPARLA 5 (2019), 119-138. 


\section{Abstract}

The study of the vocabulary pertaining to marshes and swamps among the Latin historians of the Roman Republic and of the Principatus shows that the terms used by these authors help to specify the environment of certain events - essentially military ones. The choice of these words is partly subject to the fact that in Rome, history is not a science, but a literary genre, most often at the service of an ideology or a desire for propaganda.

KEY WORDS: marsh ; swamp ; economic interest ; strategic interest ; dramatisation ; ideology ; apology 
Notre connaissance des milieux humides dans l'Antiquité repose sur des documents de quatre types: littéraires, épigraphiques, archéologiques et iconographiques. Pour ce qui est des documents littéraires latins, les chercheurs modernes se sont essentiellement attachés aux écrits des arpenteurs, agronomes, juristes, géographes, voire des poètes ${ }^{2}$. L'historiographie romaine est considérée quant à elle comme une source secondaire, voire mineure pour l'étude de ces milieux, au motif que, contrairement à ce qui se passe pour les historiens grecs, la géographie n'occupe que peu de place chez leurs homologues latins ${ }^{3}$. C'est oublier que le récit des événements rapportés par les historiens, notamment celui des opérations militaires, est indissociable du cadre dans lequel ils ont lieu. Les marais et marécages, qu'ils soient côtiers ou continentaux, sont des éléments constitutifs de ce cadre, parmi d'autres il est vrai, en particulier chez les historiens de la République et du Principat, périodes qui correspondent à la genèse de l'empire romain. Je me propose donc dans cette contribution de me pencher sur le vocabulaire latin afférant aux milieux palustres présents chez ces historiens

Les définitions des zones humides en général sont aussi nombreuses que leurs rédacteurs, qu'ils soient scientifiques, juristes, politiques ou gestionnaires. Au sens de la Convention de Ramsar, «les zones humides sont des étendues de marais, de

\footnotetext{
2 Exemples: J. Peyras, «La gestion des riparia dans l'Empire romain dans l'Antiquité tardive : les points de vue de l'arpenteur et de l'agronome », Riparia 0, 2014, p. 73-97; E. HERmon, «Sémantique, droit et pratiques agrimensorales pour la représentation spatiale des riparia ", dans E. HERMON (dir.), RIPARIA dans l'empire romain pour la définition du concept, Oxford, BAR International Series 2066, 2010, p. 231-244; R. COMPATANGELO-SOUSSIGNAN, "La représentation des embouchures fluviales dans la tradition géographique à partir du texte de Strabon", dans RIPARIA dans l'empire romain..., p. 165-176; R. BEDON, «Les rives de la Moselle dans l'œuvre d'Ausone : une illustration idéalisée de la gestion intégrée des bords de l'eau ", dans RIPARIA dans l'empire romain..., p. 191-200.

3 Exemple à propos de Tite-Live: P.G. Walsh, Livy. His Historical Aims and Methods, Cambridge, Cambridge University Press, 1961 (réimpr. 1970), p. 153-154.
} 
fagnes, de tourbières ou d'eaux naturelles ou artificielles, permanentes ou temporaires, où l'eau est stagnante ou courante, douce, saumâtre ou salée, y compris des étendues d'eau marine dont la profondeur à marée basse n'excède pas six mètres $»^{4}$. Selon le code de l'environnement français, plus restrictif, les zones humides sont en revanche des «terrains, exploités ou non, habituellement inondés ou gorgés d'eau douce, salée ou saumâtre de façon permanente ou temporaire; la végétation, quand elle existe, y est dominée par des plantes hygrophiles pendant au moins une partie de l'année $»^{5}$. Si les deux définitions divergent sur certains points, elles ont cependant un point commun, à savoir que les milieux humides ${ }^{6}$ englobent les marais et marécages.

Donner une définition des marais et marécages en français n'est guère plus aisé. Selon le Trésor de la Langue Française, le marais se définit comme un «terrain, généralement de vaste étendue, recouvert en permanence d'une nappe d'eau peu profonde, où croissent en abondance des plantes aquatiques et parfois des arbres »; le marécage est quant à lui considéré comme un « terrain spongieux, saturé d'eau, impropre à la culture, où s'étendent des marais, et qui est d'accès difficile en raison d'une dense végétation

${ }^{4}$ Convention relative aux zones humides d'importance internationale particulièrement comme habitats des oiseaux d'eau, Ramsar 2.2.1971 telle qu'amendée par le protocole de Paris du 3.12.1982 et les amendements de Regina du 28.5.1987, article Premier, alinea 1.

${ }^{5}$ Code de l'environnement, Art. L.211-1.

6 En raison de la confusion possible entre le terme français «zone humide » et les grandes zones humides définies par les climatologues à l'échelle de la planète ou encore la notion de zonage en aménagement du territoire (www.zones-humides.org/entreterre-et-eau/une-zone-humide-c-est-quoi/milieux-humides-ou-zones-hulides-0 $)$, nous utiliserons systématiquement le terme "milieu humide» dans ce qui suit. A comparer avec la définition donnée par A. BAZOGE, D. LACHANCE et C. Villeneuve, Identification et délimitation des milieux bumides du Québec méridional, Québec, Ministère du Développement durable, de l'Environnement et de la Lutte contre les changements climatiques, juillet 2004, p. 1 : «Le terme de milieu humide est utilisé pour désigner un large spectre d'écosystèmes ayant pour caractéristique commune de posséder une dynamique écologique liée à la présence de l'eau dans les sols ».

«Le vocabulaire des marais et marécages dans l'historiographie latine de la...» 
aquatique verticale $»^{7}$. Marais et marécages y sont identifiés selon le critère de l'hydrologie et de la végétation; ils sont également perçus du point de vue de l'utilisateur: le marais est présenté comme un milieu où règne une abondance de végétaux, alors que le marécage est considéré comme un espace à la fois stérile et impénétrable. On ne sera pas étonné de constater que l'importance de l'eau et de la végétation est également mise en évidence dans les définitions données par les écologistes : «Marais - Site dominé par une végétation herbacée (émergente, graminoïde ou latifoliée) croissant sur un sol minéral ou organique. Les arbustes et les arbres, lorsqu'ils sont présents, couvrent moins de $25 \%$ de la superficie du milieu. Le marais est généralement rattaché aux zones fluviales, riveraines et lacustres, le niveau variant selon les marées, les inondations et l'évapotranspiration. Un marais peut être inondé de façon permanente, semi-permanente ou temporaire »; «Marécage - Site dominé par une végétation ligneuse, arbustive ou arborescente (représentant plus de 25\% de la superficie du milieu) croissant sur un sol minéral de marais ou de très mauvais drainage. Le marécage riverain est soumis à des inondations saisonnières ou est caractérisé par une nappe phréatique élevée et une circulation d'eau enrichie de minéraux dissous. Le marécage isolé, quant à lui, est alimenté par des eaux de ruissellement ou par des résurgences de la nappe phréatique $»^{8}$. Ces exemples de définition seraient incomplets si on n'y ajoutait pas celle d'un géographe, en l'occurrence celle de P. Donadieu, pour qui « le marais désigne un terrain couvert en permanence d'une eau peu profonde »" Si les définitions présentent des divergences, une chose est sûre : on

7 P. ImBS (dir.), Trésor de la Langue Française. Dictionnaire de la langue du XIXe et du XXe siècles (1789-1960), tome 11, Paris, Klincksieck, 1985, s.v. «marais » et "marécage », p. 357 et 382.

8 A. Bazoge, D. Lachance et C. Villeneuve, Identification et délimitation des milieux humides du Québec méridional..., p. 10 et 11.

9 P. Donadieu (dir.), Paysages de marais, Paris, J.P. de Monza, 1996, p. 27. 
divise généralement les marais en marais intérieurs et marais maritimes ; enfin certains ont été drainés ou asséchés.

Le développement qui précède montre que la question des termes utilisés en français pour désigner marais et marécages, de même que celle des notions que ces mots recouvrent, ne sont pas tranchées. Il nous servira néanmoins de point de départ pour appréhender la question dans la Rome antique, et plus particulièrement dans l'historiographie latine de la République et du Principat.

Un premier constat s'impose. Le latin ne possède pas de termes différenciant "marais » et "marécages », pas plus que le grec. Deuxième constat: alors que le grec dispose essentiellement de deux vocables, ह̌ $\lambda$ o $\varsigma$ et $\lambda i ́ \mu v \eta^{10}$, pour désigner ces milieux humides, le latin ne dispose grosso modo que d'un seul terme, palus, -udis (fém.), et ses variantes, ager paluster (-tris), locus palestris, palustria (loca), dont la racine est celle qui figure dans le lituanien pilù, pilti « verser », arménien helum « je verse » et ololem "j'inonde ${ }^{11}$. De là la définition très générale de palus donnée par le Thesaurus Linguae Latinae: Significatur fere locus stagno, limo sim. occupatus siue barundinetis, iunctis uel caeno inuius siue navibus aptus ${ }^{12}$. Seule l'étude du contexte paysager, s'il existe, permet de voir éventuellement si palus désigne un marais ou un marécage. C'est ainsi que le milieu palustre du territoire d'Héraclée Trachinienne

10 Cf. Th. Chatelain, La Grèce antique et ses marais. Perception et exploitation des milieux palustres chez les Anciens, thèse de Doctorat en Lettres, Neuchâtel (Suisse) - ParisSorbonne, 2009, consultable sur www.academia.edu, p. 29-96, et plus spécialement p. 95 (synthèse sous forme de tableau : 1. ع̌ $\lambda \mathrm{o} \varsigma$ : a) environnement terrestre ; b) ensemble perçu comme un «milieu» constitué d'éléments composites (humidité, faune, végétation etc.) ; le ع̌ $\lambda \mathrm{o} \varsigma$ est un «monde» en soi ; c) frontières floues; limites indistinctes; 2. $\lambda \dot{i} \mu v \eta$ : a) environnement aquatique; b) surface aquatique perçue comme un « espace »; c) frontière visuellement close).

11 A. Ernout et A. Meillet, Dictionnaire étymologique de la langue latine, Paris, Klincksieck, retirage de la 4e édition, augmentée d'additions et de corrections par J. ANDRE, 2001, s.v. palus, p. 478.

12 ThLL X.1, s.v. palus col. 177, 1. 32-34.

«Le vocabulaire des marais et marécages dans l'historiographie latine de la... » 
doit être assimilé à un marécage car il est associé à de "grands arbres »-proceri arbores $-{ }^{13}$, alors que les " herbes des marais » - palustres herbae - et les « buissons et broussailles » - uirgulta et uepres - qui bordent le ruisseau coulant à l'ouest de la Trébie, entre le camp carthaginois et la rivière ${ }^{14}$, font plutôt penser à un paysage de marais si on s'en tient à la définition du ThLL ou à celle d'A. Bazoge, D. Lachance et C. Villeneuve.

Les textes de l'historiographe romaine, de Caton l'Ancien, premier Romain à avoir écrit une œuvre historique en latin, les Origines, jusqu'à Tite-Live, en passant par Calpurnius Pison, Sisenna, César ${ }^{15}$ et Salluste, s'accordent à mettre en relief deux caractéristiques de ce que le latin désigne par palus: a) sa situation : des «zones basses »-infima loca- ou « des vallées » - conualles - "dans lesquelles le manque de pente rend(ait) difficile l'écoulement des eaux »-ex planis locis haud facile euehebant aquas $-{ }^{16}$; b) la présence d'eau stagnante; cette présence peut être permanente : on parle alors de palus perpetua à l'exemple de ce qu'on trouve au livre VII du Bellum Gallicum de César, à propos d'un milieu palustre qu'on a identifié avec la

13 Liv. 36.22.10 : ager Heracleensis paluster omnis frequensque proceris arboribus : « le territoire d'Héraclée, tout couvert de marécages et riche en grands arbres ».

${ }^{14}$ Liv. 21.54.1 : Erat in medio riuns [...] circa obsitus palustris herbis et quibus inculta ferme uestiuntur, uirgultis uepribusque : "Il y avait dans l'espace qui les séparait, un ruisseau [...] dont le pourtour était recouvert d'herbes des marais et de la végétation propre, en général, aux lieux incultes, buissons et broussailles ».

15 César n'est pas un historien au sens propre du terme. Il est cependant rangé parmi les historiens dans les manuels de littérature latine car les Commentaires sont «le seul récit suivi et chronologiquement composé des événements qu’ils rapportent » : cf. J.-M. ANDre et A. Hus, L'bistoire à Rome, Paris, PUF, 1972, p. 28. Pour l'originalité du commentaire par rapport à l'bistoria, cf. J.-M. ANDRE et A. Hus, L'bistoire à Rome, p. 2829, et plus récemment D.L. NOUSEK, "Genres and Generic Contaminations: The Commentarii », dans L. Grillo et Chr.B. KreBs (eds), The Cambridge Companion to the Writings of Julius Caesar, Cambridge, Cambridge University Press, 2018, p. 97-109.

16 Liv. 1.38.6, à propos de Rome. Même mention de la localisation « basse » des marais ou marécages par exemple chez César: Caes. BG 3.49.4 : loca demissa ac palustria: «endroits bas et marécageux ». Sauf mention contraire, toutes les traductions des auteurs anciens seront empruntées à la CUF (Belles Lettres, Paris). 
vallée marécageuse de l'Essone, sur la rive droite de la Seine ${ }^{17}$; elle peut aussi être temporaire : une plaine boueuse se transforme ainsi en marécage par l'effet des pluies d'hiver, comme dans le cas de celui qui s'étendait autour des murailles de Suthul ${ }^{18}$; son niveau enfin, lorsqu'il s'agit d'un milieu palustre fluvial, est tributaire de la crue du fleuve, tel l'Arno qui avait inondé les marais de l'Étrurie au moment où Hannibal les avait traversés au printemps 217 pour rejoindre Arrétium : il voulut «prendre par le plus court, à travers un pays de marais inondé par une crue récente de l'Arno » ${ }^{19}$; le paysage est alors « recouvert par les eaux », au point qu' il n'y a pas un seul endroit sec où coucher pour dormir $»^{20}$.

Le marais ou le marécage peut être côtier. C'est le cas du fragment 141 Chassignet (= FRHist 26.142) de Sisenna : Sic in eam paludem multi piscium salliendorum causa a nauibus semionustis commeant ${ }^{1}$, où nous sommes peut-être en présence d'un palus situé dans un delta; il en va de même du passage livien qui, à propos

17 Caes. BG 7.57.4 : Is (sc. Camulogenus) cum animaduertisset perpetuam esse paludem, quae influeret in Sequanam... : «Ayant observé l'existence d'un marais continu qui déversait ses eaux dans la Seine... ». Autre exemple de milieu palustre permanent : celui de la ville de Pella, présentée par Tite-Live en 44.46 .5 comme entourée de «marais d'une profondeur qui les rend infranchissables en été comme en hiver»: cingunt paludes inexsuperabilis altitudinis aestate et bieme; sur la (les) rivières(s) qui alimentai(en)t ce marais : cf. J. Briscoe, A Commentary on Livy. Books 41-45, Oxford, Oxford University Press, 2012, p. 606-607.

18 Sall. Ing. 37.4 : Circum murum [...] planities limosa hiemibus aquis paludem facerat: « Autour de ses murailles (...) s'étendait une plaine boueuse transformée en marécage par les pluies d'hiver ». L'emplacement de cette ville est inconnu ; elle se trouvait probablement dans les environs de Guelma.

${ }^{19}$ Liv. 22.2.1 : Hannibal [...] propriorem uiam per paludes petit, qua flunius Arnus per eos dies solito magis inundauerat (trad. A. FLOBERT, Tite-Live, Histoire romaine : livres 6-10, Paris, GF, ut passim pour le livre 22).

${ }^{20}$ Liv. 22.2.8 : cum omnia obtinentibus aquis nibil ubi in sicco fessa sternerent corpora inueniri posset.

21 «Ils sont nombreux à aller ainsi de leurs navires à moitié chargés jusqu'à ce marais pour saler les poissons ». J. BRISCOE, dans T.J. CORNELL (dir.), The Fragments of the Roman Historians (abrégé FRHist), 3 vol., Oxford, Oxford University Press, 2013, III, p. 146, a proposé d'identifier ce marais avec un marais (marais salant) du delta du Pô.

«Le vocabulaire des marais et marécages dans l'historiographie latine de la... » 
de la ville d'Anxur, fait «descendre la ville vers les marais »Anxur [...] urbs prona in paludes -, identifiés aux marais de l'embouchure de l'Ufens (aujourd'hui l'Ufente). D'autres passages renvoient clairement à des paludes lacustres; Tite-Live en fournit deux exemples: le marais Boebeis - palus Boebeis ${ }^{22}$ - en Thessalie, de nos jours le lac de Boebeis, et le marais Labeatis palus Labeatis ${ }^{23}$-, appelé un peu plus loin par le Padouan lacus Labeatum $^{24}$, aujourd'hui le lac de Skhodra situé sur la frontière entre l'Albanie et le Monténégro ${ }^{25}$. D'aucuns ont également émis l'hypothèse que les loca palustria du fragment 31 Chassignet (= FRHist 26.25) de Sisenna correspondraient au lac Fucin ${ }^{26}$. La plupart des milieux palustres que l'on recense dans les écrits des historiens de la République et du Principat ont cependant trait à des bords de ruisseaux, rivières et fleuves, tant d'Italie que d'autres contrées. Aux rives de l'Arno et du ruisseau proche de la Trébie, vus plus haut, on ajoutera bien sûr celles du Tibre à Rome, où «nos ancêtres ne trouvaient $[\ldots]$ que bois et marais » ${ }^{27}$, la vallée de la Miette évoquée par César à propos de la bataille de l'Aisne et qualifiée par l'auteur de «marais de peu d'étendue »palus non magna ${ }^{28}$-, le ru de Berne qui sépare les camps romain et gaulois lors de l'affrontement de César avec les Bellovaques et qui est appelé palus par Hirtius ${ }^{29}$, ou encore le ruisseau, identifié par certains avec l'Arroyo de Calderón, qui, selon l'auteur du Bellum Hispanense, coulait au bas de la plaine de Munda et « débordait

\footnotetext{
22 Liv. 31.41.4.

${ }^{23}$ Liv. 44.31.3.

24 Liv. 44.31.10.

25 Cf. J. Briscoe, A Commentary on Livy. Books 41-45, p. 565.

26 Quod loca palustriaque erant et alte multifariam confossa: «Parce que les lieux étaient à la fois marécageux et creusés profondément en maints endroits ». Pour d'autres, le passage pourrait évoquer la zone marécageuse autour d'Acerrae. Sur ces différentes hypothèses d'identification de ces loca palustria, voir M. CHASSIGNET, L'annalistique romaine, t. II, texte établi et traduit par M. CHASsignet, Paris, Belles Lettres, CUF, 2004, p. 59 n. 2.

${ }^{27}$ Liv. 5.53.9 : cum in his locis nibil praeter siluas paludesque esset...

${ }^{28}$ Caes. BG 2.9.1.

${ }^{29}$ Caes. $B G$ 8.7.4 ; 8.10.2 ; 8.13.1.
} 
[...] sur la rive droite, marécageuse et pleine de fondrières $»^{30}$. Enfin il est possible que des canaux présents à Alexandrie aient présenté un caractère marécageux si on suit l'interprétation de J. Andrieu pour identifier le marais - palus - qui échancrait largement la ville d'Alexandrie au sud selon le chapitre 1 du Bellum Alexandrinum ${ }^{31}$.

Faut-il en déduire que les termes palus et paluster renvoient systématiquement aux réalités que sont les marais et les marécages et qu'inversement tout ce qui touche aux milieux palustres est évoqué par les mots palus et paluster? La réponse est négative. Nous avons vu plus haut que les paludes Boebeis et Labeatis sont en fait des lacs - laci -; de la même façon ce qu'on appelle habituellement lacus Curtius est présenté par Cornélius Pison comme un locus palustris ${ }^{32}$. Quant au mot palus qui figure dans le

30 Bell. Hisp. 29.2 : palustri et uoraginoso solo currens erat ad dextrum. Sur cette identification, cf. N. Diouron, ad loc., Paris, Belles Lettres, CUF, 1999, p. 121. Ces débordements avaient notamment lieu par temps de pluie.

31 Bell. Alex. 1.4 : palus a meridie interiecta: "un marécage interposé au sud (sc. de la ville) ». Partant de Strab. 17.792, qui précise que le lac Maréotis (aujourd'hui le lac Mariout) était alimenté par le Nil grâce à de multiples canaux se trouvant sur sa partie nord et ses côtés, J. ANDRIEU, ad loc. p. LVI, propose l'interprétation suivante : «Pourquoi ne pas envisager que le lacis de ces cours d'eau formait une région marécageuse qui débordait par le sud dans un quadrilatère de la ville, et que coupait le canal du Nil à Alexandrie coulant en direction de l'Euroste ? Cette disposition répondrait aux indications du Bellum Alexandrinum, à la dépression observée sur ce terrain, et à la manœuvre de César (sc. rejoindre le marais, fortifier ses flancs et, son but atteint, couper Alexandrie en deux)».

32 Calp. Piso frg. 8 Chassignet (= 9.8 FRHist) à propos de l'étymologie « sabine » du lac Curtius : Piso in Annalibus scribit Sabino bello, quod fuit Romulo et Tatio [...] Metrium Curtium Sabinum $[. .$.$] in locum palustrem, qui tum fuit in foro antequam cloacae sunt factae, secessisse atque$ ad suos in Capitolium recepisse; ab eo lacum inuenisse nomen: "Pison dans ses Annales écrit que pendant la guerre sabine qui opposa Romulus et Tatius [...], le Sabin Mettius Curtius se retira dans un endroit marécageux qui s'étendait sur le forum avant la construction des égouts et rejoignit les siens au Capitole. C'est de lui que le lac a tiré son nom ». Pour une description du bassin découvert à son emplacement, voir par exemple F. COARELLI, Il foro Romano. I. Periodo arcaico ${ }^{2}$, Rome, Quasar, 1992, p. 50-52. Même mention d'un marais - palus - dans la version "sabine» rapportée en Liv. 1.12 .10 (sans mention de sa source). Dans la version "romaine» de la denotio de M. Curtius, il est question en revanche d'« un vaste gouffre - specus uastus - d'une immense profondeur » ou encore d' " un abîme » - norago - : cf. Liv. 7.6.1 et 2.

«Le vocabulaire des marais et marécages dans l'historiographie latine de la...» 
fragment 141 Chassignet de Sisenna, il désigne peut-être un marais salant, généralement appelé salina ${ }^{33}$. Inversement la plaine marécageuse d'Almajar, située au nord de Carthagène, est désignée par Tite-Live par le vocable stagnum, alors qu'à propos du même lieu, Appien parle d'Ě $\lambda$ os et Polybe de $\lambda \hat{\mu} \mu v \eta^{34}$.

La mention des marais et marécages est bien évidemment inséparable de celle d'autres termes inhérents à leur évocation. En premier lieu la présence de la boue et de la vase par le biais du substantif limus : les troupes d'Hannibal y ont été confrontées lors de leur traversée des marais de l'Étrurie, « enfonçant dans la vase - limus - où ils risquaient de se noyer $»^{35}$; on rencontre également cette boue sur le site de la bataille des Thermopyles qui a eu lieu en 191 ; Tite-Live rapporte en effet que «sur la droite des Macédoniens, juste à l'extrémité des fortifications » se trouve un «endroit où la boue des marécages - paluster limus - et les fondrières ferment jusqu'à la mer une région qu'elles rendent impraticable $»^{36}$. Le dérivé limosus apparaît quant à lui chez Salluste, dans le passage cité ci-dessus, à propos du marécage qui entourait les murailles de Suthul ${ }^{37}$. Ces marais et marécages sont

${ }^{33}$ Exemples : Caes. BC 2.37.5 : aquae et salis copia, cuius magna uis iam ex proximis erat salinis eo congesta: "l'abondance d'eau et de sel, dont une grande quantité, provenant des plaines voisines, avait été déjà rassemblée en ce point»; Liv. 1.33.9 : in ore Tiberi Ostia urbs condita, salinae circa factae : «il (sc. Ancus Marcius) fonda Ostie à l'embouchure du Tibre et fit des marais salants tout autour ».

34 App. Ib. 20 ; Pol. 10.10. Pour une définition de stagnum, cf. P.G.W. GLare, Oxford Latin Dictionary (abrégé OLD), Oxford, Oxford University Press, 1968-1982, réimpr. avec corrections 1996, 2e éd. révisée 2012, s.v. stagnum, p. 1813: 1. An expanse of standing water, pool, lagoon etc. (natural or artificial) ; 2. (applied to any expanse of water) : a. (a part of the sea); b. (of a river). Dans le cas présent, il s'agit en fait d'une lagune. Stagnum apparaît aussi chez Liv. 1.4 .2 à propos des débordements du Tibre : lenia stagna «nappes d'eau dormante ». Pour son dérivé restagnare, voir Liv. 44.46 .5 à propos des marais entourant la ville de Pella: paludes [...] quas restagnantes faciunt <amnes> (Madvig; lac. V) : «ils forment une nappe d'eau alimentée par des $<$ rivières $>$ ».

${ }^{35}$ Liv. 22.2.5 : hausti paene limo immergentesque se.

${ }^{36}$ Liv. 36.18.4 : Ab dextro Macedonibus ad ipsum munimentorum finem, qua loca usque ad mare palustri limo et uoraginibus claudunt.

37 Pour le texte, voir n. 18. 
parfois franchissables par des gués - uada -, à l'exemple de ceux qui permettaient de traverser les marais entourant pour l'un le site d'Avaricum, assiégé par César ${ }^{38}$, et pour l'autre la position occupée par les Bellovaques et leurs alliés lors de cette même guerre des Gaules ${ }^{39}$. Les paludes sont par ailleurs souvent associées à des fondrières - uoragines et leur variante uoraginosum solum - : c'est le cas pour uoragines dans l'évocation livienne, au livre 22 de l' $A b$ V rbe condita, des marais de l'Étrurie traversés par l'armée d'Hannibal ${ }^{40}$, ou encore dans celle, au livre 36, du site de la bataille des Thermopyles ${ }^{41}$, pour uoraginosum solum dans la description de la rive droite de l'Arroyo de Calderón qui figure dans le Bellum Hispanense ${ }^{42}$. Enfin, un certain nombre de passages font état de la végétation, poussant soit dans les paludes proprement dites, soit à proximité immédiate de ces milieux humides. Aux «herbes des marais»-palustres herbae -, les «buissons et broussailles »- uirgulta et uepres - et les " grands arbres »-proceri arbores - déjà vus à propos de la distinction entre marais et marécages ${ }^{43}$, on ajoutera les «fourrés 》- saltus et les «forêts »- siluae $-{ }^{44}$. Pour ce qui est d'une mention éventuelle de la faune caractéristique des paludes, sauf erreur de notre part, elle est inexistante.

Si les termes recensés jusqu'ici sont communs à l'évocation des marais et marécages dans l'ensemble de la littérature latine, il va de soi que le point de vue de l'auteur n'est pas le même selon qu'il

38 Caes. BG 7.19.1 : <Galli> omnia uada ac saltus eius paludis obtinebant: « les Gaulois occupaient solidement tous les gués et tous les fourrés ».

${ }^{39}$ Caes. BG 8.13.1 : ... proelia [...] quae ad uada transitusque fiebant paludis : « combats [...] qui ont lieu aux endroits guéables du marais ».

40 Liv. 22.2.5.

${ }^{41}$ Liv. 36.18.4. Pour le texte, voir n. 36.

42 Bell. Hisp. 29, 2. Pour le texte, voir n. 30.

43 Pour le texte, voir n. 13 et 14.

44 Saltus: Caes. BG 7.19.1 à propos du site d'Avaricum : pour le texte, voir n. 38 ; siluae : Caes. BG 3.28.2 à propos des Morins et des Ménapes : quod [...] continentes [...] siluas ac paludes habebant: "possédant une région que couvraient sans interruption forêts et marécages »; Liv. 5.53.9 (pour le texte, voir n. 26).

«Le vocabulaire des marais et marécages dans l'historiographie latine de la...» 
s'agit d'un écrit poétique, agronomique, encyclopédique ou historiographique. Dans le cas de l'historiographie en général et celle de la République et du Principat en particulier, ce point de vue peut varier selon les passages. Trois points de vue se dégagent des textes recensés.

$\checkmark$ point de vue «hygiéniste ${ }^{45}$. Dans ce cas de figure, l'auteur met l'accent sur le climat malsain d'une ville comme Gravisques, « ainsi nommée parce qu'un air lourd - grauis aer - pes< $<$ ait $>$ sur elle ${ }^{46}$, ou explique la perte de l'usage d'un œil d'Hannibal lors de la traversée des marais d'Étrurie par les veilles, l'humidité des nuits mais aussi par «les vapeurs - umor - qui montent des marais et alourdissent la tête palustre caelum grauans caput $»^{47}$.

point de vue économique. L'historien, au détour du récit, indique quel avantage économique peuvent offrir les paludes. Les marais d'eau salée permettent de saler les poissons - pisces sallire-, qui ont été pêchés ailleurs, à condition d'y pénétrer avec des bateaux à moitié chargés $^{48}$. Ce milieu palustre peut être par ailleurs parfois aménagé en marais salants : c'est ce qu'aurait fait Ancus Marcius puisque, selon la tradition rapportée par TiteLive, il « fonda Ostie à l'embouchure du Tibre et fit des marais salants - salinae - tout autour $»^{49}$. Enfin, lorsqu'il

\footnotetext{
${ }^{45}$ L'adjectif est emprunté à Th. CHATELAIn, La Grèce antique et ses marais, p. 140.

46 Cat. Orig. frg. II, 17 Chassignet (= 5.71 FRHist). La ville de Gravisques, colonie romaine fondée en 181 à l'emplacement de l'ancien port étrusque de Tarquinia, était connue pour son climat malsain, insalubre - intempestus, pestilens - ; elle périclita an raison du caractère marécageux de la côte. Cf. M. CHAssignet : Caton: Origines (fragments), texte établi et traduit par M. CHAssignet, Paris, Belles Lettres, CUF, 1986 (2e tirage 2002), p. 74, n.1 ad loc.

${ }^{47}$ Liv. 22.2.11.

48 Sisenna frg. 141 Chassignet (= FRHist 26.142). Pour le texte, voir supra, n. 21.

${ }^{49}$ Liv. 1.33.9 : in ore Tiberis Ostia urbs condita, salinae circa factae. Sur la date effective de la création de ces marais-salants, voir R.M. OGILvie, A Commentary on Livy. Books 1-5, Oxford, Clarendon Press, 1965, p. 140.
} 
est asséché, le marais ou le marécage offre une surface urbanisable comme dans le cas de Rome ; toujours selon la tradition, les «quartiers bas vers le forum et dans les vallées » auraient été asséchés - siccare - par Tarquin l'Ancien grâce à " un système d'égouts allant des points élevés vers le Tibre $»^{50}$.

$\checkmark$ point de vue stratégique. Une partie non négligeable des écrits historiographiques ayant par la force des choses pour sujet des opérations militaires, on ne sera pas étonné de constater que bon nombre de passages font grand cas de l'influence des milieux palustres sur le déroulement des opérations. Marais et marécages sont, selon le cas, un obstacle difficile à franchir pour un assaillant ou au contraire un lieu faisant office de protection ou encore offrant ressources en eau et en bois à ceux qui en sont démunis.

L'A V V rbe condita en fournit des exemples. Selon le Padouan, l'armée d'Hannibal, partie à la poursuite de Flaminius, n'a franchi les marais de l'Étrurie qu'au prix de lourdes pertes, tant humaines que matérielles: soldats qui sont morts «au milieu des bêtes de somme étendues de tous côtés »; "hommes épuisés d'avoir dû se passer de sommeil pendant quatre jours et trois nuits »; impossibilité "dans ce paysage recouvert par les eaux», de «trouver un seul endroit sec pour dormir »; Hannibal qui «finit par perdre l'usage d'un œil en raison des veilles, de l'humidité des nuits, des vapeurs qui montent des marais et alourdissent la tête et faute de trouver un endroit ou moment pour se soigner $»^{51}$. Aux

${ }^{50}$ Liv. 1.38.6. Pour la construction des égouts mais sans datation, voir aussi Calp. Piso fr. 8 Chassignet (= 9.8 FRHist) : pour le texte, voir n. 31. Sur la chronologie de l'assèchement de cette partie de la Ville, cf. R.M. OGILvie, A Commentary on Livy. Books 1-5, p. 156.

51 Liv. 22.2, 7-11 : inter iumenta et ipsa iacentia passim morientes; maxime omnium vigiliae conficiebant per quadriduum iam et tres noctes toleratae; cum omnia obtinendis aquis nibil ubi in sicco «Le vocabulaire des marais et marécages dans l'historiographie latine de la...» 
Thermopyles, la boue des marécages et les fondrières rendent la région située sur la droite du camp Macédonien «impraticable »inuia ${ }^{52}$; a contrario lors du siège d'Héraclée, le territoire de la cité, "tout couvert de marécages et riche en grands arbres", a offert aux Romains «en abondance du bois pour tous les travaux ", à savoir la construction " de tours, de béliers et toutes les autres machines de siège $»^{53}$.

Si Tite-Live fournit des exemples des effets de l'existence de marais et marécages sur le déroulement des événements militaires, c'est incontestablement l'ensemble du corpus césarien qui est le plus riche pour notre examen du vocabulaire relatif à cette thématique dans l'historiographie de la République et du Principat. Pour ce qui est de l'obstacle qu'ils représentent, on relèvera essentiellement deux termes : l'adjectif difficilis et son dérivé difficultas, et impedio, principalement présent sous sa forme d'adjectif verbal impeditus, verbe dont le sens étymologique «empêcher de marcher» apparaît pleinement ici ${ }^{54}$. La position occupée par les Gaulois à Avaricum est entourée de toutes parts d'un marais «difficile à traverser » - difficilis - et "plein d'obstacles» - impedita $-{ }^{55}$. Confronté aux Bellovaques, César, "voyant qu'entre les deux camps (sc. gaulois et romain) s'étendait un marais qui formait un obstacle sérieux - impedita - et capable d'empêcher une poursuite rapide - quae difficultas celeritatem insequendi tardare posset - », devra faire appel à ses qualités de stratège pour surmonter cette difficulté ${ }^{56}$. Impeditus qualifie

fessa sternerent corpora inueniri posset; Hannibal [...] nigiliis [...] et nocturno umore palustrique caelo granante caput et medendi nec locus nec tempus erat altero oculo capitur.

52 Liv. 36.18.4.

53 Liv. 36. 22.10 : Et cum ager Heracleensis paluster omnis frequensque proceris arboribus benigne ad omne genus operum materia suppeditabat; ... turres arietesque et alius omnis apparatus oppugnandarum urbium.

54 A. ERnout et A. Meillet, Dictionnaire étymologique de la langue latine, s.v. pes, p. 501.

${ }_{55}$ Caes. BG 7.19 .1 : palus difficilis atque impedita.

${ }^{56}$ Caes. BG 8.14.4 : cum palude impedita a castris castra diuidi uideret, quae transeundi difficultas celeritatem insequendi tardare posset. 
également les marais du pays des Éburons ${ }^{57}$; quant à Camulogène, il se félicite de l'existence d'un marais «qui rendait l'accès de toute la région (sc. à proximité de Lutèce) fort difficile » - impedire $-{ }^{58}$. Marais et marécages peuvent constituer un obstacle pour les uns; ils sont inversement susceptibles de faire office d'abri ou de protection pour les autres. C'est ainsi que le « marais d'accès difficile »-palus impedimenta-- du pays des Éburons offre à ces mêmes indigènes un espoir de protection - praesidium ou de salut - salus - face aux Romains ${ }^{59}$. Quant aux Bellovaques et leurs alliés, qui ont établi leur camp au milieu d'un bois entouré d'un marais, ils bénéficient eux aussi de «la protection du marais ", la notion de protection étant cette fois-ci exprimée par le déverbatif munire, emprunté au vocabulaire militaire ${ }^{60}$. Enfin les milieux palustres peuvent offrir eau et fourrage à des combattants qui en sont dépourvus : les Pompéiens coupés par César de leur approvisionnement en eau près de Dyrrachium en sont réduits à "chercher les endroits bas et marécageux » et à y creuser «des puits »-putei - pour pallier le manque d'eau ${ }^{61}$; lors du siège d'Alexandrie, le marais interposé au sud de la ville peut pour sa part offrir «largement» à César eau et fourrage - aqua pabulumque - à un moment où il ne dispose plus que d'une faible quantité de l'une et manque totalement de l'autre ${ }^{62}$.

Ces mentions correspondent-elles à la réalité ? La première question que l'on est en droit de se poser est celle des sources. Si

\footnotetext{
57 Caes. BG 6.34.2 : palus impedita.

${ }^{58}$ Caes. BG 7.57.4 : quae [...] illum omnem locum magnopere impediret.

${ }^{59}$ Caes. BG 6.34.2 : Vbi cuique [...] palus impedita spem praesidii aut salutis aliquam offerebat, consederat: "Partout où [...] un marécage d'accès difficile offrait quelque espoir de protection ou de salut, on y avait cherché asile».

${ }^{60}$ Caes. BG 8.11.1 : Caesar, cum animaduerteret hostem complures dies castris palude [...] munitis se tenere... : «César, voyant que les jours passaient et l'ennemi restait dans son camp sous la protection d'un marais... ». Pour le rapport moenia/ munire, voir A. ERNOUT et A. MEILLET, Dictionnaire étymologique de la langue latine, s.v. moene; moenia, p. 410.

${ }^{61}$ Caes. BC 3.49.4.

62 Bell. Alex. 1.5 : utrumque (sc. aquam pabulumque) large palus praebere poterat: « le marécage pouvait lui fournir largement les deux ».
}

«Le vocabulaire des marais et marécages dans l'historiographie latine de la...» 
pour les passages recensés chez Caton, Calpurnius Pison, Sisenna et Salluste, il n'est guère possible de se prononcer sur ce sujet, il n'en va pas de même pour le corpus césarien et pour Tite-Live. Les sources du Padouan, on le sait, sont purement livresques : Tite-Live a repris et mis en forme un certain nombre de récits antérieurs, notamment ceux des annalistes, sans citer forcément ses sources. L'exemple le plus patent pour ce qui concerne notre propos est le passage consacré à la version sabine de l'étymologie du milieu palustre appelé palus/lacus Curtius, d'abord attestée chez Calpurnius Pison puis mentionnée, uniquement pour mémoire il est vrai, dans l' $A b V$ rbe condita ${ }^{63}$. César et ses continuateurs, quant à eux, ont essentiellement puisé leur matière dans les rapports d'état-major rédigés par ses légats, ainsi que des rapports que César envoyait lui-même, périodiquement, au Sénat pour justifier son action et se faire prolonger dans ses fonctions ${ }^{64}$; il s'agit donc de documents reposant sur des expériences dont César a été l'acteur. À cette documentation s'ajoutent des développement géographiques et ethnographiques, sans doute d'origine livresque mais le plus souvent non identifiée. Il va sans dire que ces sources livresques ou l'expérience personnelle ne sauraient être un gage de l'impartialité de l'évocation. L'histoire à Rome n'est pas une science, mais un genre littéraire, que ce soit celui de l'œuvre historique au sens strict du terme ou de Commentaires. De là la recherche de la dramatisation dans l'Ab Vrbe condita dans le célèbre passage qui a pour sujet la traversée des marais de l'Étrurie : si Tite-Live y évoque les marais de l'Arno, l'épisode lui permet, grâce à ce procédé de l'exornatio, de peindre un tableau au souffle épique incontestable et d'apporter en même temps une touche au portrait d'Hannibal. D'autres passages du Padouan sont au contraire l'occasion de mettre en avant son patriotisme, en idéalisant les soldats et les grands hommes de Rome, à l'image

63 Voir supra n. 31. Tite-Live privilégie de fait la version «romaine » qui fait de M. Curtius un Romain et non un Sabin (épisode de la deuotio).

${ }^{64}$ Cf. M. Rambaud, L'art de la déformation historique dans les Commentaires de César, Paris, Belles Lettres, 1966 (1ère éd. 1952), passim. 
d'un Ancus Marcius créateur des marais salants d'Ostie. De là aussi une évocation très "orientée » des milieux palustres chez César: si les marais et les espaces marécageux sont présentés comme un obstacle sur sa route, c'est pour mieux mettre en évidence les qualités de l'auteur des Commentaires qui expose volontiers son action lorsqu'elle permet de résoudre un problème et de la justifier. L'ennemi en revanche est incapable de tirer profit de l'atout dont il dispose.

Au total donc, des lexèmes qui touchent à tous les domaines des milieux palustres, à l'exception de la faune. Leur étude montre qu'ils servent logiquement à préciser le cadre dans lequel se déroulent certains événements, essentiellement militaires. Une analyse un peu plus poussée permet de voir que le choix de ces vocables est en partie subordonné au fait que l'histoire à Rome n'est pas une science mais un genre littéraire, le plus souvent au service d'une idéologie ou d'une volonté de propagande. 


\section{Bibliographie}

J.-M. ANDRÉ, A. Hus, L'bistoire à Rome, Paris, PUF, 1972.

J. ANDRIEU: Bellum Alexandrinum, texte établi et traduit par J. ANDRIEU, Paris, Belles Lettres, CUF, 1954, 3e tirage 2002.

A. BAzOGE, D. LACHANCE, C. VILlENEUVE, Identification et délimitation des milieux bumides du Québec méridional, Québec, Ministère du Développement durable, de l'Environnement et de la Lutte contre les changements climatiques, juillet 2004.

R. BEDON, «Les rives de la Moselle dans l'œuvre d'Ausone: une illustration idéalisée de la gestion intégrée des bords de l'eau ", dans E. HERMON (dir.), RIPARIA dans l'empire romain pour la définition du concept, Oxford, BAR International Series 2066, 2010, p. 191-200.

J. BRISCOE, A Commentary on Livy. Books 41-45, Oxford, Oxford University Press, 2012.

M. CHASSIGNET : Caton: Origines (fragments), texte établi et traduit par M. Chassignet, Paris, Belles Lettres, CUF, 1986 (2e tirage 2002).

M. CHASSIGNET, L'annalistique romaine, t. II, texte établi et traduit par M. Chassignet, Paris, Belles Lettres, CUF, 2004.

Th. Chatelain, La Grèce antique et ses marais. Perception et exploitation des milieux palustres chez les Anciens, thèse de Doctorat en Lettres, Neuchâtel (Suisse) - Paris-Sorbonne, 2009, consultable sur www.academia.edu. F. COARELl, Il foro Romano. I. Periodo arcaico ${ }^{2}$, Rome, Quasar, 1992. R. COMPATANGELO-SOUSSIGNAN, «La représentation des embouchures fluviales dans la tradition géographique à partir du texte de Strabon », dans E. Hermon (dir.), RIPARIA dans l'empire romain pour la définition du concept, Oxford, BAR International Series 2066, 2010, p. 165-176.

T.J. CORNELL (dir.), The Fragments of the Roman Historians (abrégé FRHist), 3 vol., Oxford, Oxford University Press, 2013.

N. Diouron: Bellum Hispanense, texte établi et traduit par N. Diouron, Paris, Belles Lettres, CUF, 1999, 2e tirage 2002.

P. Donadieu (dir.), Paysages de marais, Paris, J.P. de Monza, 1996.

A. ERnout, A. MEILLET, Dictionnaire étymologique de la langue latine, Paris, Klincksieck, retirage de la 4 e édition, augmentée d'additions et de corrections par J. ANDRÉ, 2001.

P.G.W. GLARE, Oxford Latin Dictionary (abrégé OLD), Oxford, Oxford University Press, 1968-1982, réimpr. avec corrections 1996, 2e éd. révisée 2012. 
E. HERMON, «Sémantique, droit et pratiques agrimensorales pour la représentation spatiale des riparia », dans E. HERMON (dir.), RIPARIA dans l'empire romain pour la définition du concept, Oxford, BAR International Series 2066, 2010, p. 231-244.

P. IMBS (dir.), Trésor de la Langue Française. Dictionnaire de la langue du XIXe et du XX' siècles (1789-1960), tome 11, Paris, Klincksieck, 1985.

D.L. NOUSEK, «Genres and Generic Contaminations: The Commentarii », dans L. Grillo et Chr.B. Krebs (eds), The Cambridge Companion to the Writings of Julius Caesar, Cambridge, Cambridge University Press, 2018, p. 97-109.

R.M. OGILvie, A Commentary on Livy. Books 1-5, Oxford, Clarendon Press, 1965.

J. PEYRAS, «La gestion des riparia dans l'Empire romain dans l'Antiquité tardive : les points de vue de l'arpenteur et de l'agronome », Riparia 0, 2014, p. 73-97.

M. RAMBAUD, L'art de la déformation historique dans les Commentaires de César, Paris, Belles Lettres, 1966 (1 ìre éd. 1952).

Thesaurus Linguae Latinae (abrégé ThLL), tome XI.1, Leipzig, Teubner, 1984 (version online Berlin, De Gruyter).

P.G. WAlsh, Livy. His Historical Aims and Methods, Cambridge, Cambridge University Press, 1961 (réimpr. 1970), p.153-154.

«Le vocabulaire des marais et marécages dans l'historiographie latine de la...» 